\section{Orales Allergiesyndrom: Respirationstrakt reagiert nicht mit}

\begin{abstract}
Atemwege und oberer Verdauungstrakt haben einen gemeinsamen embryonalen Ursprung. Dies lässt die Überlegung zu, dass die Atemwege im oralen Provokationstest beim oralen Allergiesyndrom mitreagieren könnten.
\end{abstract}

talienische Wissenschaftler wollten herausfinden, ob beim oralen Allergiesyndrom auch der Respirationstrakt auf eine orale Provokation hin reagiert. Hierzu wurden vier Probandengruppen gebildet:

-Gruppe A: an Asthma bronchiale und Rhinitis allergica leidende Birkenpollenallergiker mit oralem Allergiesyndrom (Apfel);

_ Gruppe B: Birkenpollenallergiker mit oralem Allergiesyndrom ohne Asthma bronchiale oder Rhinitis allergica;

_Gruppe C: Birkenpollenallergiker mit Asthma bronchiale und Rhinitis allergica ohne begleitendes orales Allergiesyndrom;

_Gruppe D: gesunde Probanden.
Allen Probanden wurde roher Apfel in ansteigender Dosis bis zu einer Höchstmenge von $128 \mathrm{~g}$ fraktioniert zugeführt. Diese Mindestdosis wurde benötigt, um bei den Birkenpollenallergikern mit bekanntem oralen Allergiesyndrom eine orale Symptomatik auszulösen. Vor und nach den Versuchen wurden die klinischen Symptomatik notiert, Lungenfunktionstests ausgewertet und ausgeatmetes Stickoxid sowie die Eosinophilen im Nasenausstrich bestimmt. Die Untersuchungen wurden in der pollenflugfreien Zeit durchgeführt. Alle Patienten waren zur Zeit der Untersuchung asymptomatisch und medikationsfrei.

Nach der oralen Provokation kam es in den Gruppen A und B erwartungsgemäß zur Entwicklung eines oralen Allergiesyn- droms. Reaktionen des Respirationstraktes konnten hingegen nicht registriert werden.

Die Probanden der Gruppe C entwickelten weder eine orale Symptomatik, noch wurden die Lungenfunktionsparameter beeinflusst.

Die gesunde Kontrollgruppe D blieb symptomenfrei und die Tests unverändert normal.

Fazit: Birkenpollenallergiker mit oralem Allergiesyndrom infolge einer Kreuzreaktion mit Apfel haben nach Verzehr von Äpfeln keine Reaktion des Respirationstraktes zu befürchten. Dies muss aber nicht für jedes kreuzreagierende Nahrungsmittel, nicht einmal für eine andere Apfelsorte gelten. Pollenallergiker sollten kreuzreagierende Nahrungsmittel daher besser meiden oder nur inaktiviert genießen.

Dr. Dieter Bruchhausen

Lombardi et al. The functional connection between oral allergy syndrome and united airways disease assessed by oral challenge. Ann Allergy Asthma Immunol 2012;108: 30-3

\section{Was bringt die Interleukin-5-Hemmung bei eosinophiler Ösophagitis}

\begin{abstract}
Die Therapie der eosinophilen Ösophagitis ist vor allem bei Kindern und Jugendlichen problematisch. Denn die üblichen Glukokortikoide können unter anderem das Wachstum verzögern. US-Forscher haben nun versucht herauszufinden, ob den Betroffenen mit dem Interleukin-5-Hemmer Reslizumab zu helfen ist. Ergebnis: teils, teils.
\end{abstract}

E r kenntnisse aus Tierversuchen legen nahe, dass Interleukin 5 (IL-5) die eosinophile Entzündung im Ösophagus befeuert. Reslizumab ist ein monoklonaler Antikörper, der in der Lage ist, IL-5 zu neutralisieren. Daher könnte sich mit der Substanz auch der Verlauf der eosinophilen Ösophagitis beeinflussen lassen.

An einer entsprechenden randomisierten, doppelt verblindeten und placebokontrollierten Studie beteiligten sich 226 Patienten im Alter von fünf bis 18 Jahren. Rund 90\% von ihnen wiesen klinisch mäßige bis sehr schwere Symptome auf, wie etwa Schmerzen, Erbrechen oder Schluckbeschwerden. Im Median waren in den Gewebeproben der Speiseröhre
80 intraepitheliale Eosinophile pro Hauptgesichtsfeld (400-fache Vergrößerung, 0,3 $\mathrm{mm}^{2}$ ) zu finden. $\mathrm{Zu}$ Beginn der Studie sowie nach vier, acht und zwölf Wochen erhielten die Probanden 1, 2 oder $3 \mathrm{mg} / \mathrm{kg}$ Reslizumab oder eine Placebolösung als Infusion. Nach 15 Wochen wurden die Gewebseosinophilen erneut gezählt. Außerdem wurde der klinische Befund erhoben und anhand des „Physician's Eosinophilic Esophagitis Global Assessment" einem von fünf Graden zugeordnet.

Tatsächlich hatte die Zahl der Eosinophilen bei jenen Probanden, die den IL5-Hemmer infundiert bekommen hatten, im Vergleich zu Placebo signifikant abgenommen. Die Reduktion betrug $59 \%$ in der $1-\mathrm{mg}$-, $67 \%$ in der 2 -mg- und $64 \%$ in der 3-mg-Gruppe. Auch klinisch ergaben sich Verbesserungen: Hatten zu Studienbeginn nur knapp 10\% der Teilnehmer keine oder leichte Beschwerden, waren es nun $56,4 \%$ ( $1 \mathrm{mg}$ ), 63,2\% (2 $\mathrm{mg}$ ), 64,9\% (3 mg) bzw. 71,9\% (Placebo). Die Verumsubstanz schnitt hier aber nicht signifikant besser ab als Placebo.

Fazit: Reslizumab wirkt gegen eosinophile Ösophagitis von Kindern und Jugendlichen - zumindest unter dem Mikroskop. Klinisch bringt eine zwölfwöchige Intervalltherapie zunächst keine Vorteile gegenüber Placebo. $\mathrm{Ob}$ eine längerfristige Therapie besser abschneidet, wird zurzeit untersucht.

Dr. Robert Bublak

Spergel JM et al. Reslizumab in children and adolescents with eosinophilic esophagitis: Results of a double-blind, randomized, placebo-controlled trial. J Allergy Clin Immunol 2012; 129: 456-63 